\title{
El proceso contable y su normativa
}

\section{The accounting process and its regulations}

Yolanda Tatiana Carrasco Ruano. ${ }^{1}$, María Auxiliadora Falconí Tello. ${ }^{2}$, Mauricio Chávez Haro. ${ }^{3} \&$ Silvia Guadalupe Naranjo Lozada. ${ }^{4}$

\section{DOI: https://doi.org/10.33262/visionariodigital.v5i4.1914}

\begin{abstract}
The evolution of technology and science over time is undeniable, and laws and regulations are not left out of it. Regulations evolve with the actions of people, constantly changing and adapting to the environment to avoid becoming obsolete and keep up with new forms of business, increasingly influenced by a global economy, where the greatest good is knowledge. Objectives: this work aims to identify the current accounting regulations within the accounting process. Methodology: it applies the quantitative method, bibliographic design, documentary type, cross-sectional, analytical explanatory.
\end{abstract} Contribution: establish a documentary base of the current accounting regulations.

Keywords: IAS, IFRS, processes, accounting, standards.

\section{Resumen}

La evolución de la tecnología y la ciencia a través del tiempo es inegable, y las leyes y normativas no se quedan fuera de ella. La normativa evoluciona con el accionar de la gente, constantemente cambia y se adapta al medio para evitar quedar en deshuso y seguirle el paso a las nuevas formas de negocios, cada vez más influenciados por una economía global, donde el mayor bien es el conocimiento. Objetivos: el presente trabajo

${ }^{1}$ Ciencia Digital, investigador independiente, Ambato, tcarrascor@yahoo.com. ORCID https://orcid.org/. 0000-0002-5294-8202

${ }^{2}$ Escuela Superior Politécnica del Chimborazo ESPOCH, Facultad de Administración de Empresas, auxiliadora.falconi@espoch.edu.ec. ORCID https://orcid.org/.0000-00029014-2912

${ }^{3}$ Escuela Superior Politécnica del Chimborazo ESPOCH, Facultad de Ciencias Pecuarias, Riobamba, mauricio.chavez@espoch.ec, ORCID https://orcid.org/.0000-0003-1335-6885

4 Universidad Técnica de Ambato, Facultad de Contabilidad y Auditoría, dranaranjosil@gmail.com, ORCID https://orcid.org/.0000-0003-4559-4060 
pretende identificar la normativa contable vigente dentro del proceso contable. Metodología: aplica el método cuantitativo, diseño bibliográfico, tipo documental, de corte transversal, analítico explicativo. Aporte: establecer una base documental de la normativa contable actual.

Palabras claves: NIC, NIIF, procesos, contable, normas.

\section{Introducción}

La evolución de la tecnología y la ciencia a través del tiempo es innegable, y las leyes y normativas no se quedan fuera de ella. La normativa evoluciona con el accionar de la gente, constantemente cambia y se adapta al medio para evitar quedar en deshuso y seguirle el paso a las nuevas formas de negocios, cada vez mas influenciados por una economía global, donde el mayor bien es el conocimiento.

Este articulo pretende identificar la normativa vigente dentro del proceso contable, como se involucra en cada uno de los procesos y subprocesos. La NIIF establece procedimientos clara e inequívocos de como proceder ante distintos casos. Esta normativa es actualizada anualmente y constantemente está siendo revisada.

El conjunto de normas contables que están aceptadas y son usadas en la gran mayoría de países son emitidas por "The International Accounting Standard Committee" (IASC), cuya sede se encuentra en Inglaterra y fue creado en el año de 1973. Este organismo es el responsable de la emisión y actualización continua de la normativa contable (Toledo et al., 2021)

Todo el proceso contable involucra al contador, evidenciado los hechos económicos conforme a su perfil lo exige, para asegurar que la información se presente de manera confiable, fiable, oportuna y siempre reflejando la realidad de la actividad económica (Albero et al, 2021).

A nivel de Latinoamérica las NIC (Normas Internacionales de Contabilidad) - NIIF (Normas Internacionales de Información Financiera), se aplicaron desde el año 2009 (A. Fierro y F. Fierro, 2016).

Al no estar claro como intervienen las normas en el proceso contable, debido a la gran cantidad de información que existe para aplicar, el presente trabajo pretende una guía rápida para poder aplicar las NIC - NIIFS, identificando con claridad las normas básicas en cualquier tipo de actividad económica (Toledo et al., 2021)

\section{Metodología}

La presente investigación aplica el método cuantitativo, diseño bibliográfico, tipo documental, de corte transversal, analítico explicativo con los objetivos del estudio por el método de causa efecto, sin intervención del investigador. La justificación del problema realiza un enfoque teórico, al analizar la normativa actual y su participación dentro del proceso contable. 
La justificación del problema, indica la razón por la cual se quiere realizar dicha investigación, y el aporte que se pretende lograr en los distintos ámbitos, sean estos: sociales, políticos, económicos, humanos, etc. (Palella y Martins, 2012, p. 60).

\section{Marco Teórico}

Como se presenta en el trabajo de Toledo et al. (2021), la información financiera parte de una Hipótesis Fundamental, antes de la aplicación de cualquier norma, es necesario que los estados financieros se preparen en base a las siguientes hipótesis:

- Devengo: los efectos de las transacciones se reconocen cuando ocurren, independientemente de cuando se realice su cobro o su pago,

- Empresa en funcionamiento: esta hipótesis supone que la empresa está en funcionamiento y continuara sus actividades operativas dentro de un futuro previsible.

Las NIIF se han convertido en el lenguaje global de facto de la información financiera, que se utiliza ampliamente en las economías desarrolladas, emergentes y en vías de desarrollo (NIIF, 2021).

Los procesos contables dentro de una organización, expresan las políticas, procedimientos, controles; disminuyendo los desaciertos y presentando razonabilidad a los estados financieros (Valdez et al., 2019, pág. 2).

Los procedimientos económicos de una entidad para la presentación de estados financieros, forman parte del proceso contable de la institución, son actividades que la ser identificadas en un proceso rutinario establecen las políticas para los procesos (Barreto y Teodomiro, 2018).

Toda la información emitida bajo la normativa NIIF, está enfocada a emitir estados financieros para la economía mundial, y la ayuda en la toma de decisiones en las finanzas corporativas y la discusión sobre la colocación de inversiones, que intentan colocar recursos financieros escasos tratando de obtener un equilibrio entre rentabilidad, riesgo y liquidez (Macedonio y García, 2020).

Pero toda esta toma de decisiones se verá afectada por el método de valoración que el profesional contable selecciono para la preparación de los estados financieros. Las empresas generalmente utilizan el coste histórico; este se combina con otras bases de valoración de cuentas específicas. Además, algunas empresas usan el coste corriente como respuesta a la incapacidad del modelo contable del costeo histórico (Amat y Puig, (2018).

En la tabla 1, se muestra la clasificación de los principales grupos de los estados financieros y sus métodos de valoración. 
ISSN: 2602-8506

\section{Tabla 1}

Métodos de valoración de los elementos de los estados financieros

\begin{tabular}{|c|c|c|}
\hline Método & Activos & Pasivos \\
\hline $\begin{array}{l}\text { Coste Histórico } \\
\text { (Valor de adquisición) }\end{array}$ & $\begin{array}{l}\text { Se registran por el importe de } \\
\text { efectivo y otras partidas } \\
\text { pagadas, o por el valor } \\
\text { razonable, de la contrapartida } \\
\text { entregada a cambio en el } \\
\text { momento de la adquisición. }\end{array}$ & $\begin{array}{l}\text { Se registro por el valor del producto } \\
\text { recibido a cambio de incurrir en la } \\
\text { deuda o, en algunas circunstancias } \\
\text { (por ejemplo, en el caso de los } \\
\text { impuestos), por las cantidades de } \\
\text { tesorería y otros medios líquidos } \\
\text { que se espera pagar para satisfacer } \\
\text { la correspondiente deuda en el } \\
\text { curso normal de la operación. }\end{array}$ \\
\hline $\begin{array}{l}\text { Coste Corriente } \\
\text { (Valor que constaría en la } \\
\text { actualidad) }\end{array}$ & $\begin{array}{l}\text { Se registran por el importe de } \\
\text { tesorería y otros medios } \\
\text { líquidos, que deberían pagarse } \\
\text { si se adquiriese en la } \\
\text { actualidad el mismo activo u } \\
\text { otro equivalente. }\end{array}$ & $\begin{array}{l}\text { Se registran por sus valores de } \\
\text { liquidación, es decir por los } \\
\text { importes sin descontar de tesorería } \\
\text { u otros medios líquidos, que se } \\
\text { espera que puedan satisfacer las } \\
\text { deudas en el curso normal de la } \\
\text { operación. }\end{array}$ \\
\hline $\begin{array}{l}\text { Valor Realizable } \\
\text { (Valor de liquidación actual) }\end{array}$ & $\begin{array}{l}\text { Se registra el valor presente, } \\
\text { descontando las entradas netas } \\
\text { de efectivo que se espera que } \\
\text { genere la partida en el curso } \\
\text { normal de la operación. }\end{array}$ & $\begin{array}{l}\text { Se registra por el valor presente, } \\
\text { descontando las salidas netas de } \\
\text { tesorería que se espera necesitar } \\
\text { para pagar las deudas en el curso } \\
\text { normal de la operación. }\end{array}$ \\
\hline
\end{tabular}

Fuente: Vazquez et al. (2013)

Una de las normas generales fundamentales es la NIIF 13, medición del valor razonable. En esta norma se hace alusión a cuatro métodos para la valoración contable de transacciones

- Costo histórico, de acuerdo a como ocurrió en el momento de realización de la transacción.

- Costo corriente, registro contable según el valor actual o en el presente.

- Valor realizable, equivale a lo que podría obtenerse en el mercado en el momento de su valuación.

- Valor presente, monto actual descontado el efectivo percibido desde el inicio de su existencia hasta su liquidación (NIIF, 2021).

En la figura 1 se muestra la jerarquización de las normas: están las normas generales que se aplicaran en todas las cuentas de un estado financiero y prevalecerán sobre las normas específicas, que solo aplican a determinadas cuentas.

En la parte mas alta de la pirámide esta la norma mas importante, el "marco conceptual", mismo que establece entre otras cosas el objetivo de la información financiera a revelar, características de la información, elementos de los estados financieros y su definición (Activo, Pasivo, Patrimonio), reconocimiento y baja de cuentas, bases de medición. 
En resumen el marco conceptual ayuda comprender las normas e interpretar las normas.

\section{Figura 1}

Categorización de las NIIF

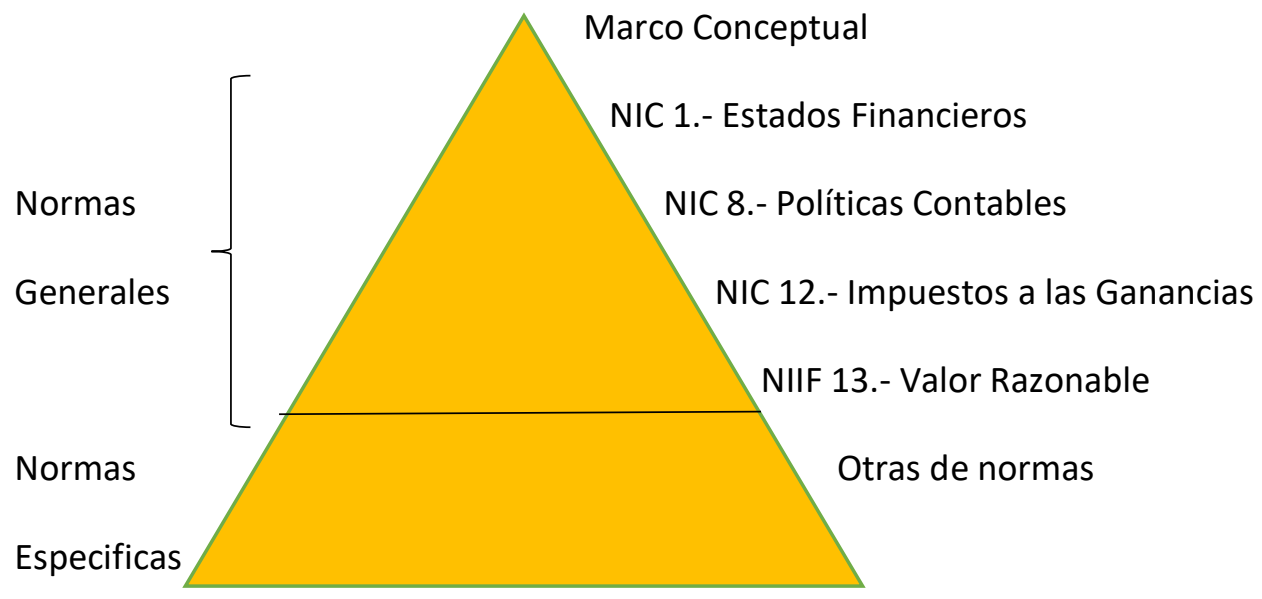

Nota: Las normas generales se aplican en todos los tipos de actividades económicas y supeditan las normas específicas

Fuente: Toledo et al. 2021

En el trabajo de Toledo et al. (2021), se categoriza la normativa, y proponen un flujo del proceso contable como se muestra en la figura 2 , donde se establecen cinco momentos, que son:

1. Identificación

2. Reconocimiento

3. Medición

4. Presentación

5. Revelación

Cada uno de los momentos del proceso contable, esta normado por una NIIF o NIC, según el caso. Esta normativa establecerá el método de valuación de cada cuenta, así como las políticas contables, controles y registros, para su posterior presentación en los estados financieros o revelaciones en sus notas (Toledo et al., 2021). 
ISSN: 2602-8506

\section{Figura 2}

\section{Proceso Contable normativa NIIF}

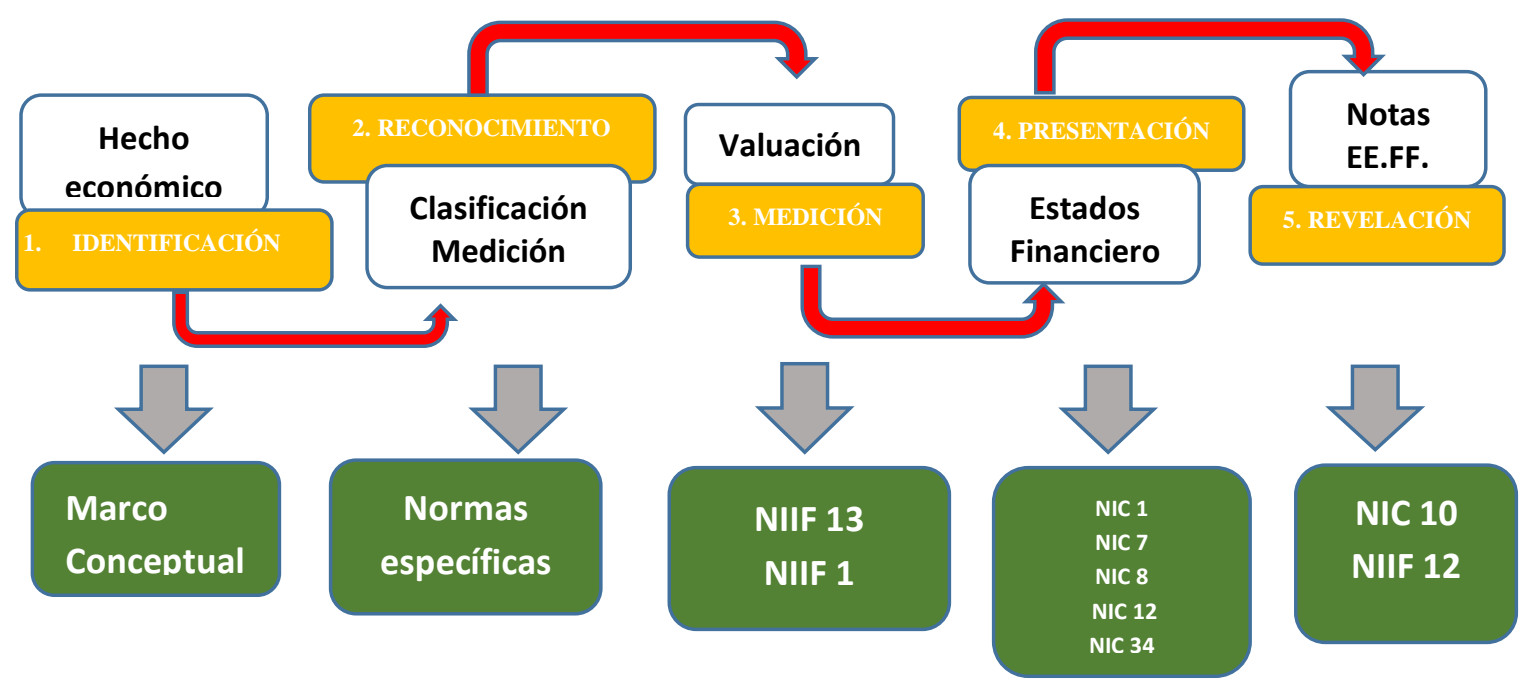

Nota: Las normas generales se encuentran presente en todo momento del proceso contable.

Fuente: Toledo et al. (2021)

En la figura 3 se plasma un proceso particular de una norma específica para el control de la cuenta de activos biológicos.

\section{Figura 3}

Proceso contable de activos bilógicos normativa NIIF

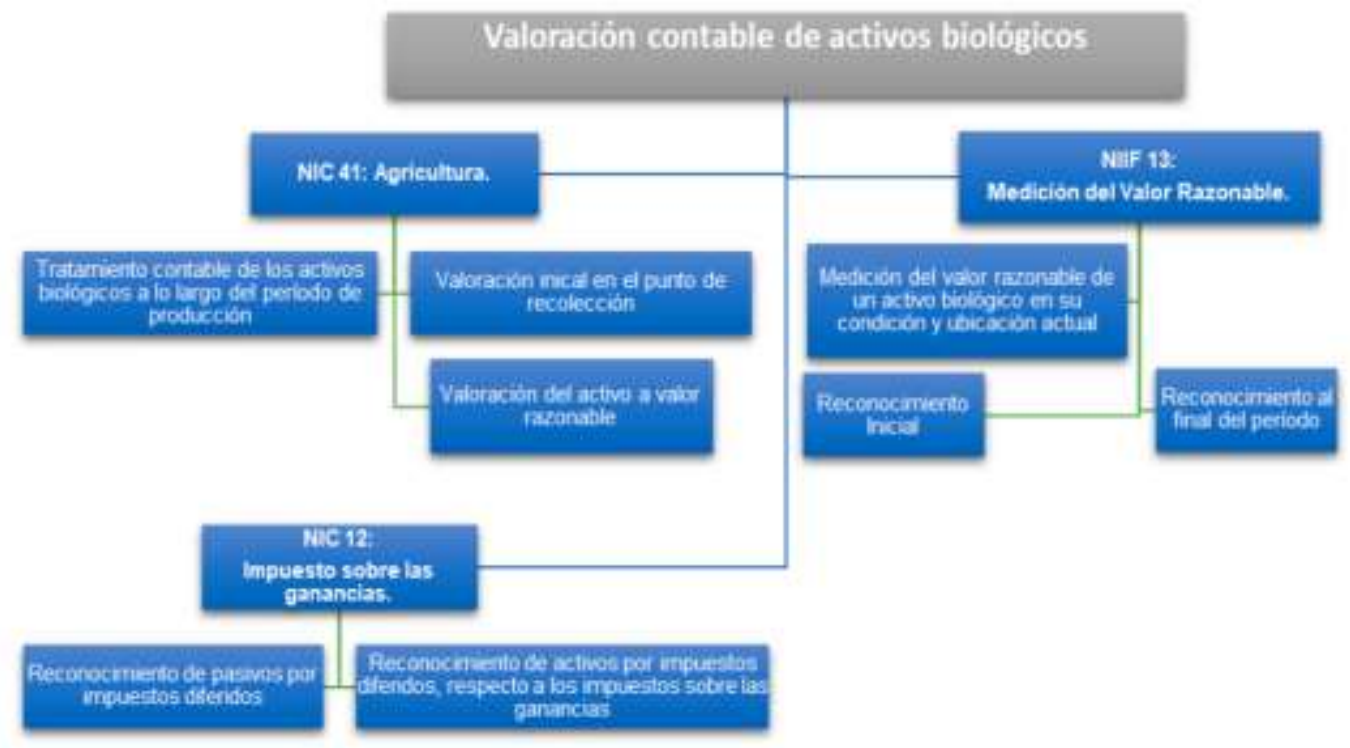

Nota: Las diversas partidas contables, generan procesos contables específicos según la normativa NIIF.

Fuente: Reyes et al, 2019.

Como se observa en la figura 3, la norma específica va acompañada en su proceso contable de las normas generales, para que exista uniformidad en la aplicación de las 
políticas contables y coherencia en la presentación de los estados financieros (Toledo et al., 2021).

\section{Discusión}

Como se pudo observar los procesos específicos son varios, uno por cada Norma Internacional de Contabilidad (NIC), o por cada Norma Internacional de Información Financiera (NIIFF). En el marco teórico se presento algunos ejemplos de procesos contables bajo normativa NIIF.

Como aporte al trabajos ya presentados se presentan los procesos contables de la NIC 2 de inventarios, cuyo alcance no incluye las obras en curso (NIC 11 contratos de construcción), los instrumentos financieros (NIC 32 y NIC 39), activos biológicos (NIC 41 agricultura), las materias primas cotizadas en bolsa. El proceso contable de todos los inventarios, excepto los antes mencionado se describe en la figura 4.

\section{Figura 4}

\section{Proceso Contable Inventarios NIC 2}

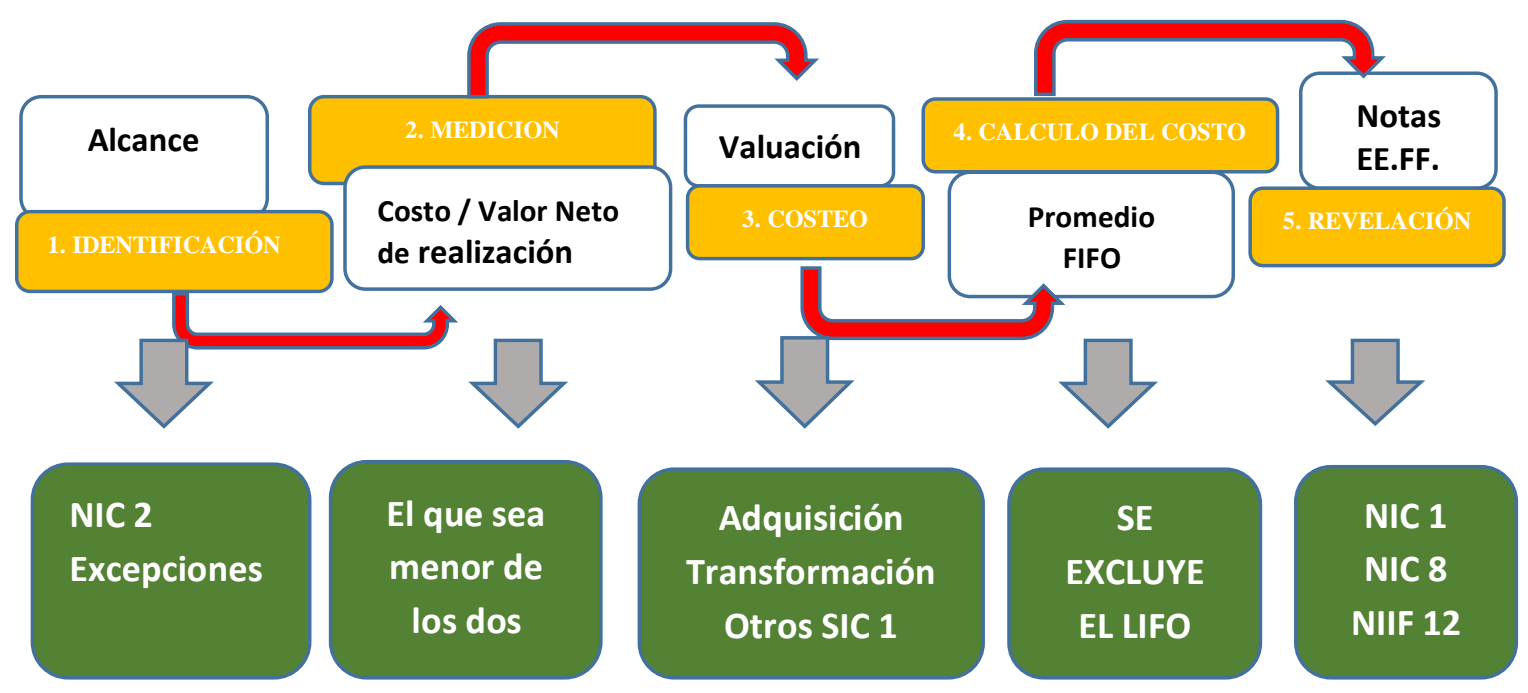

El proceso contable descrito en la figura 4, sobre inventarios (NIC 2), muestra el proceso y su normativa contable, desde el alcance de la norma, sus exclusiones, hasta la decisión de ser presentados en balances o simplemente revelados en notas.

Con la aplicación del proceso y su normativo, el registro de los inventarios sigue un esquema simple y claro de como debe ser presentada la información de la cuenta.

Se estableció que se generan procesos específicos para cuentas de los estados financiero, sin embargo eso no quiere decir que no se pueda establecer un proceso, para una norma general como es la NIC 1 (presentación de estados financieros). En la figura 5, se plasma el proceso para NIC 1. 
Figura 5

Proceso Contable Presentación Estados Financieros NIC 1

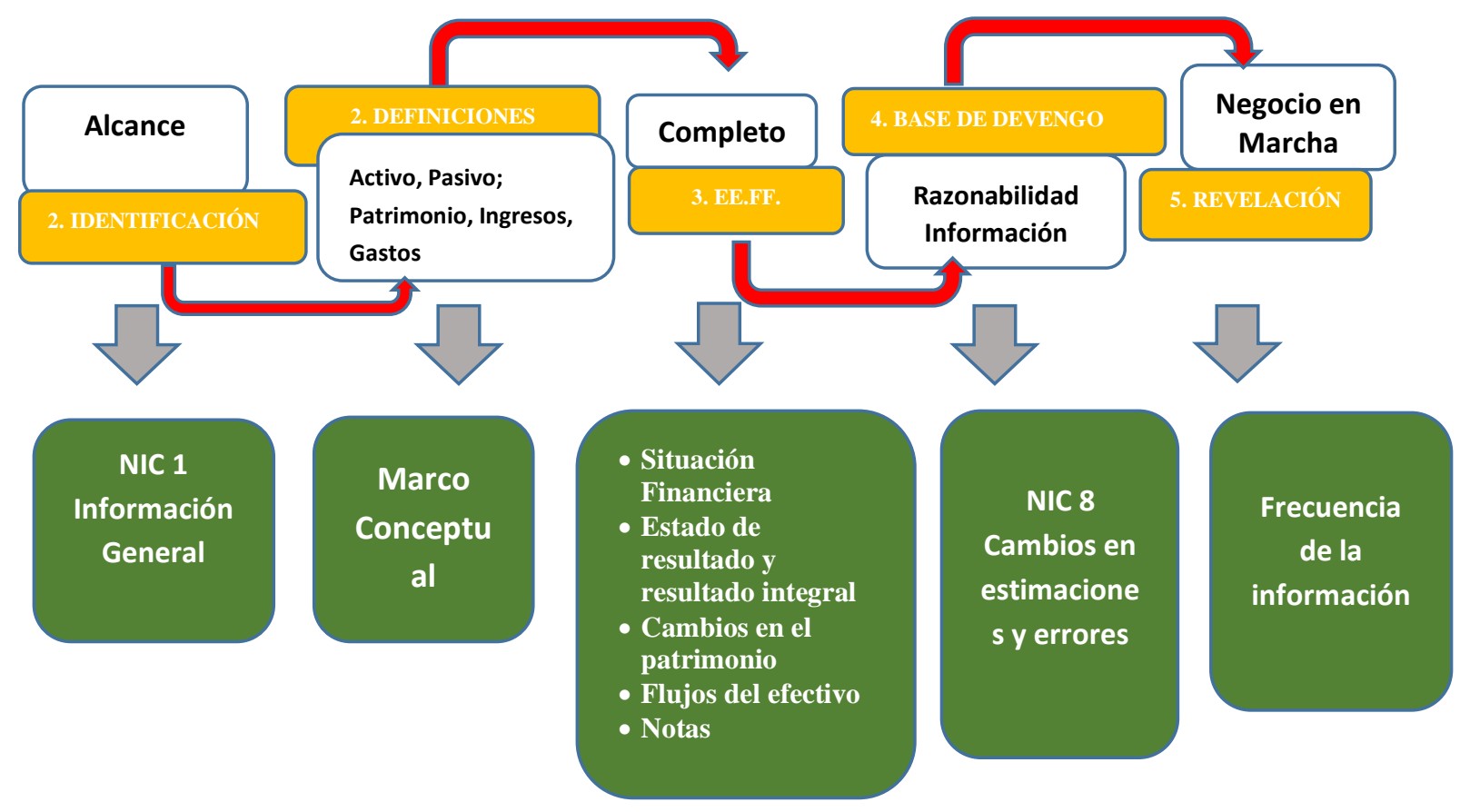

La NIC 1, al ser una norma general, establece los lineamientos y definiciones básicas para el reconocimiento de cuentas y su presentación. Establece conceptos básicos como la uniformidad de la información, al obligar a mantener las políticas contables seleccionadas a lo largo de toda la vida de una cuenta.

\section{Conclusiones}

- Antes de que el mundo entero adoptara las NIIF, existían un sin número de normativas particulares, mismas que al ser específicas y dedicadas a la legislación local, no permitían la uniformidad de la información, dificultando una clara y precisa información para sus usuarios.

- Al establecer la normativa en un esquema gráfico, es fácil apreciar lo bien pensada que fue, con procedimientos claros para su reconocimiento, medición, costeo y presentación.

- Los procesos humanos son flexibles y siempre están sujetos a cambios, evolucionan con el tiempo y sus sociedades, ajustándose en el tiempo. Son increíblemente influenciados por el comercio y todas sus formas de interacción humana, la normativa NIIF es un claro ejemplo de ello.

\section{Referencias Bibliográficas}

Albero Carballosa, C., Prado Chaviano, E., Celestrín Penabades, C., Jiménez Valero, B., \& Velastegui López, L. E. (2021). Acciones para la formación inicial de la competencia profesional específica registrar hechos económicos. Visionario Digital, 5(2), 216-224. https://doi.org/10.33262/visionariodigital.v5i2.1720 
Barreto, J. y Teodomiro E. (2018). Análisis de los procesos operacionales y contables desarrollados en la empresa. Lima: Soluciones Empresariales EIRL Trujillo.

Fierro, A. y Fierro, F. (2016). Contabilidad de activos con enfoque NIIF para las PYMES. Bogotá: Ecoe ediciones.

Macedonio, Alanís y García, Pablo (2020). Tecnología de la información y la práctica contable. ISBN-13: 979-8-6212-4443-9

Amat, Oriol y Puig, Xavier. (2018). Master en Finanzas: Claves, fundamentos, estrategias y operativas de las finanzas empresariales. Profit Editorial I.

Normas Internacionales de información Financiera NIIF (2021), https://www.ifrs.org/

Normas Internacionales de información Financiera NIIF (2021), https://www.ifrs.org/use-around-the-world/why-global-accounting-standards/

Palella Santa y Martins Feliberto. (2012). Metodología de la investigación cuantitativa (1ra reimpresión). Caracas: Fedupel ISBN: 980-273-445-4.

Reyes Arana, M. Y., Narváez Zurita, C. I., Andrade Amoroso, R. P., \& Erazo Álvarez, J. C. (2019). Valoración contable de activos biológicos bajo NIIF en la empresa camaronera Biotónico S.A. Visionario Digital,3(2.1.), 476-496. https://doi.org/10.33262/visionariodigital.v3i2.1..585

Toledo Castillo, N. del R., Peñafiel Moncayo, I. R., \& Carrasco Ruano, Y. T. (2021). Las NIC -NIIF dentro del proceso contable. AlfaPublicaciones, 3(3.1), 43-54. https://doi.org/10.33262/ap.v3i3.1.77

Valdez Fajardo, W. P., Narváez Zurita, C. I., Ormaza Andrade, J. E., \& Erazo Álvarez, J. C. (2019). Procedimientos contables y su incidencia en la sistematización de la información de la Cooperativa de Ahorro y Crédito Atlántida de la ciudad de Cañar. Visionario Digital, 3(2.1.),

497-526. https://doi.org/10.33262/visionariodigital.v3i2.1..586

Vazquez, Nitzia y Díaz, Manuel. (2013). Normas Internacionales de Informacion Financiera (NIIF-IFRS). Profit Editorial.

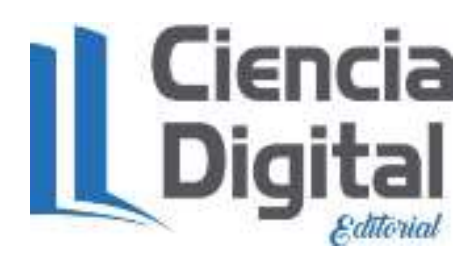




\section{PARA CITAR EL ARTÍCULO INDEXADO}

Carrasco Ruano, Y. T., Falconí Tello, M. A., Chávez Haro, M., \& Naranjo Lozada, S. G. (2021). El proceso contable y su normativa. Visionario Digital, 5(4), 78-87. https://doi.org/10.33262/visionariodigital.v5i4.1914

\section{Ciencia}

El artículo que se publica es de exclusiva responsabilidad de los autores y no necesariamente reflejan el pensamiento de la Revista Visionario Digital.

El artículo queda en propiedad de la revista y, por tanto, su publicación parcial y/o total en otro medio tiene que ser autorizado por el director de la Revista Visionario Digital.
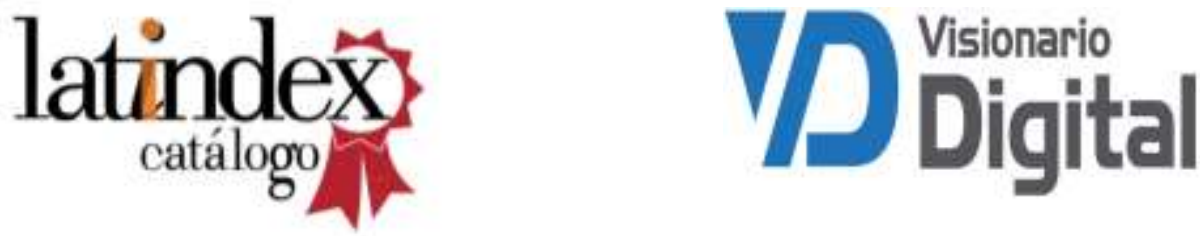